\title{
Design of a Mobile App with the Use of Machine Learning for the Monitoring of Coronavirus Patients (COVID 19) In Peru
}

\author{
Alexi Delgado ${ }^{1}$, Enrique Lee Huamaní ${ }^{2}$, VentocillaGomero- Fanny ${ }^{3}$, Morales Zarate-Edith ${ }^{3}$ \\ ${ }^{1}$ Systems Engineering Program, Pontificia Universidad Católica del Perú, Lima-Peru,kdelgadov@ pucp.edu.pe \\ ${ }^{2}$ Image Processing Research Laboratory (INTI-Lab), Universidad de Ciencias y Humanidades, Lima-Peru, \\ ehuamani@uch.edu.pe \\ ${ }^{3}$ Systems Engineering Program, Universidad de Ciencias y Humanidades, Lima-Perú, fanventocillag@ uch.pe
}

\begin{abstract}
Globally, the massive expansion of acute respiratory syndrome (COVID_19) is mainly caused by the massive agglomeration of people at the time of travel, as a person infected with the virus who does not have the respective preventive measures can infect 3 more people according to studies. For this reason, here is proposed a mobile application with the use of the Machine Learning methodology for future prediction, through the historical data learned. In this scenario, historical data collection is performed and a decision tree is designed to evaluate the behavior of the data divided into three evaluation criteria (high, medium, and low) probability. As a result, the design of the App is shown with spaces for patient follow-up through, constant chat (doctor-patient), patient communication forums, prescriptions, recommendations and up-to-date information about the virus. This app will be useful for all Peruvian citizens as they avoid mass congestion of people when they move to a health center to have a discard test or other frequently asked questions to a doctor.
\end{abstract}

Key words: Machine Learning; Mobile app; Decision tree, COVID_19.

\section{INTRODUCTION}

In Latin America, Peru was one of the first countries in which the government decrees the state of emergency, closed borders and quarantined citizens in order to control the expansion of the severe acute respiratory syndrome virus (SARS-CoV2- COVID 19), such early measures were taken due to Peru's weak health system [1], While the prevention measure has been used to buy time and better equip the health system and prevent the massive increase in the rate of contagions [2], however at the time of publication of this article the scale of contagion tends to $13.13 \%$ per 100 thousand inhabitants, thus indicating the collapse of health systems in different departments of the Peruvian state [3]. The cases of contagion have increased due to the massive increase of the inhabitants in health centers, supermarkets, Banks [4], it is sought to contribute to the reduction of the contagion rate of this social problem is designed a mobile application that is useful for the citizens of Peru.
As for methodologies for predicting future data several of them were found, and others for the design of the mobile application, as first of them we have the Holt method, to present future trends through mathematical equations that provides estimates of the slope of the trend line for the time period [5].As another methodology for predicting the Winters method is found, predictions are also made through mathematical formulas whose responses estimate between 0 and 1 it is necessary to analyze the data and create a date variable previously [6]. There is also Machine Learning (ML), which is defined as the process through which computers are programmed to record information [7] and these can be structured and unstructured which are displayed through algorithms that allow us to identify patterns in the data, allowing us to improve the compression and learning of a study topic and even to predict the behavior of a data in the future.

In the ML learning process I can be classified into two: supervised learning and unsupervised (automatic) learning. In supervised learning, the data is labeled in some way given part to the computer to feed on experimental Q \& amp ; A data, then proceeds to create a model that is adjusted to the answers obtained, the classification algorithms are what allow us to achieve the goal of this purpose [8], in this case the use of the decision tree is implemented, this being a supervised machine tilt algorithm made as a structured flowchart so that it includes a root node, sheets and decision nodes to be made, the data is continuously separated according to the parameters established, where the sheets act as decisions i.e. final results and decision nodes are used for data division and are very friendly in their usability does not require the parameter domain [[9], which will be used to facilitate the supervised machine learning approach, which will be divided into 3 categorical values for each section to improve the veracity of predictions. And as for the design of the application is Proto.io, which allows transactions of screens of the prototype so that the application is observed as if it was already implemented [10]. In addition this MOCKFLOW, which is an interactive and easy software for drawing and user-friendly interfaces for mobile applications, and is the one used for prototype design in this article. 
This article presents the design of a mobile application, to track patients (those who were discharged, asymptomatic patients and future patients) in case of future patients considers the coronavirus discard test that would be implemented in the mobile application with the Machine Learning study [11], said test would be performed through data collection (in this case the data are the symptoms presented by patients confirmed with COVID_ 19). In addition, the application will allow continuous communication between doctors and patients, including interconnection for recommendations, participation in forums and others, among registered patients, as well as information on the updated situation of coronavirus and a map of the area's most prone to contagion with the virus in order to alert the citizen.

The objective of this work is to design a mobile application to follow up discharged, asymptomatic and future patients, implementing a mobile application with Machine Learning. In this way, it will contribute to the reduction of the rate of COVID_19 infections.

The present study is organized in the following way: section II will describe the methodology to be used, section III will show the case study in the textile company Chalicen S.A.C., section IV will discuss the results obtained and finally, section $\mathrm{V}$ will present the conclusions regarding the general objective proposed.

\section{METHODOLOGY}

In example mode to train more supervised learning, it requires a large amount of data to train a classification model, and requires fairly considerable storage and processing as a mobile application (APP) system requirement. In unsupervised or automatic learning you can store your cloud models such as the mHealth APP where it allows storing patient data for remote monitoring and diagnosis, in addition to managing a patient's condition, quantitative information of a patient can also be recompiled and certain symptoms controlled, just like remote monitoring and clinical decisions could be provided to patients discharged [12]. While as seen in the example, unsupervised machine learning may have a number of advantages due to its cloud storage but it also has multiple drawbacks such as: latency, the time it takes for the service to respond to the customer request. Intimacy, problems may arise with sensitive data. Cost, to cloud service providers. Connectivity, network connection is required for availability.

That is why we will work with supervised learning as we are working with current dormant data, thus facilitating the collection of data day after day [13].

\subsection{The Process Phases of Machine Learning}

For the development of the application, Machine Learning is implemented, which has an interactive sequence as shown in figure 1, the steps to correctly analyze the mass data according to the ML are observed.

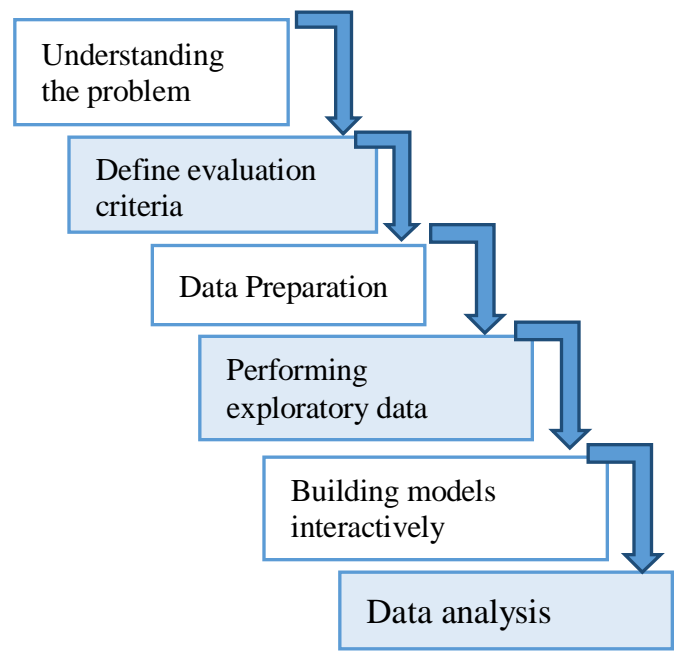

Figure 1:The Process Phases of Machine Learning

\section{A. Understanding the problem}

They meet to analyze and formulate the problem to be analyzed through why?

\section{B. Define evaluation criteria}

Regressions and cross-entropy can be used for data classification problems due to the presence of several issues that require evaluation.

\section{Data Preparation}

Significant analysis and analysis of data is performed through comparisons, combinations with various sources..

\section{Performing exploratory data and model construction} interactively

The part where data will gain intuition in relation to variables and should be related to expert judgment through agile processes with continuous improvements. And in the model construction part, where most of the time is addressed because at this stage feedback is generated and compared with other models until the project objective is met [14].

\section{E. Data analysis}

It enables scientifically supported data-driven decision-making and there are four categories of analysis that are distinguished by the results they produce [15]. Descriptive analysis: serves to answer questions about events that have already occurred. Diagnostic analysis: its goal is to determine the cause of a phenomenon that occurred in the past through questions that are focused on the reason behind the event. Predictive analysis: it is done in an attempt to determine the result of an event that could occur in the future, in this type of analysis the information improves thanks to the related information stored in the past to be improved in the future [14] as we can see in the Figre 2 below: 


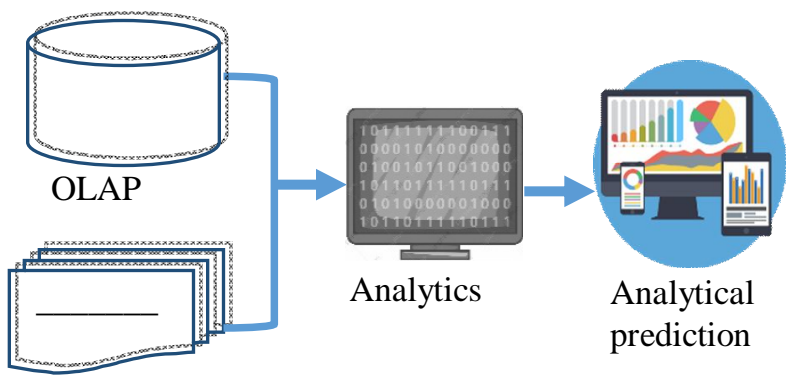

Textual

1 .

Figure 2:Predictive data analysis

\subsection{Suggested technological tools for the application}

For the operating system that the application server will have is proposed to CentOS Linux, because it is a distribution that is obtained from sources published by Red Hat for Red Hat Enterprise Linux (RHEL), in addition to being an open source operating system that offers its users enterprise-class software because it is defined as robust, easy and stable for installation and use [16].

For the exposition of the application services is proposed the use of Apache HTTP Server because it is open source for moderate operating systems, it includes UNIX and Windows, since it is also suggested that this project can provide an efficient and efficient server sicronized with the current states.

The proposed database for the application will be NoSQL as it is a moderate database that prioritizes the escability, performance and flexibility of its structures, and also allows the handling of large volumes of data and fast speed [17].

To develop the application you plan to use the Android programming language with Kotlin, since it is based on other programming languages such as java and $\mathrm{C}++$, which allows you to have an updated programming. It is used using Android Studio as it is an integrated development environment that offers features that increases productivity during the execution of the app, it also contains packages necessary for development that have support and updates for the development of new versions of Android [18].

As for the Framework, it is proposed to use Ionic or jQuery Mobil, for an interactive environment of the application, in addition to being hybridized [18]. For the design of the initial prototype presenseized in this paper will be WireframePro, as it is a tool with numerous components for pre-built packaging interfaces for iOS, Android, Web, WatchOS, Bootstrap and others [14].

\section{CASE STUDY}

With the methodologies mentioned, the detailed processes for the correct analysis will be explained below.

\subsection{The Process Phases of Machine Learning}

\section{A. Understanding the problem (coronavirus description (COVID_19))}

It is a virus that tends to cause acute and severe respiratory diseases called (SARS-CoV2- COVID 19) that was identified in Wuhan China in late 2019, this virus affects human health to the degree of mortality because they have been shown to be single-stranded RNA viruses that are easy to mutate, and are spread through breathing. The World Health Organization (WHO) declared the problem an emergency of international concern [19], this virus has been leaving high mortality rates in different countries due to its massive contagion, so many countries have had to send all their inhabitants to quarantine to prevent the spread of the virus, thus crippling the world economy.

\section{B. Define evaluation criteria}

E In the figure. 3 shows the results of the tests carried out grouped in a period of 7 days where $8.5 \%$ of samples were positive to COVID-19 [1].

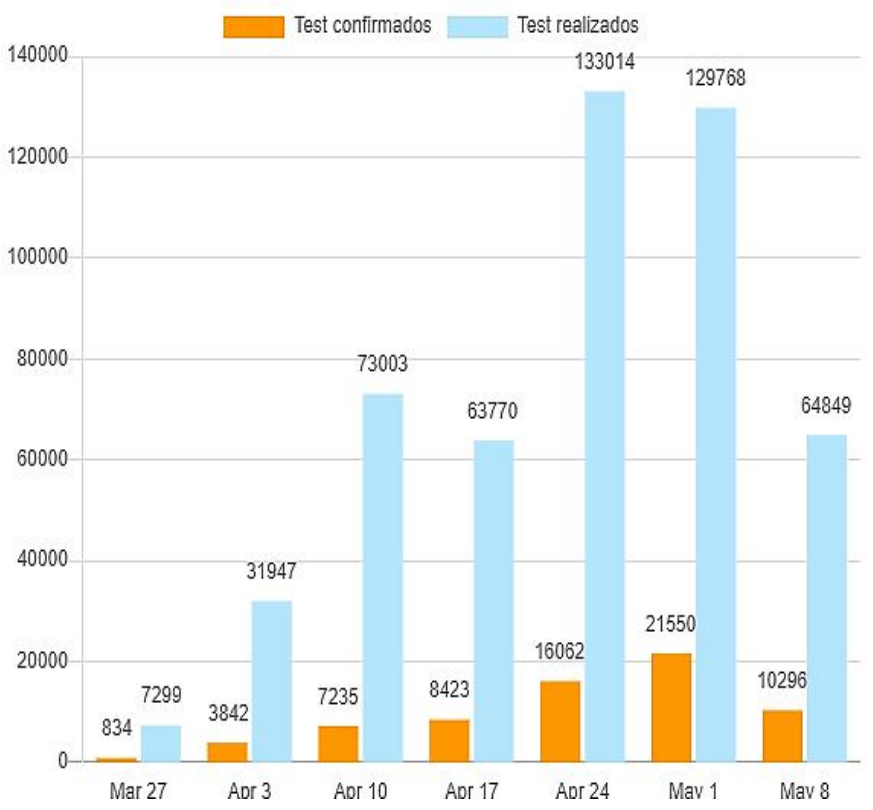

Figure 3: Tests carried out in Peru in 7 days

\section{Data preparation}

Table 1 shows the already selected data that we will use for data analysis to predict how likely in patient to re-contract COVID 19 and follow up on patients discharged for decision-making

Table 1: patient follow-up

\begin{tabular}{|l|l|}
\hline \multicolumn{2}{|c|}{ PATIENTS DISCHARGED } \\
\hline DESCRIPTION & AMOUNT \\
\hline Number of high patients & 22406 \\
\hline Cases detected & 68822 \\
\hline Deceased & 1961 \\
\hline
\end{tabular}

\section{Conducting exploratory data and building a model}

In the figure 4 we can observe the symptoms of patients detected positive with COVID_19, where it is observed that 
$72.8 \%$ of those detected had symptoms of cough, $8.8 \%$ of them had fever and chilled, $57.7 \%$ of them indicated to have general discomfort, $57.5 \%$ had a sore throat, $32.6 \%$ headache, $30.5 \%$ nasal congestion, 30.1 breathing difficulties, $21.8 \%$ muscle pain symptoms, 12.9 diarrhoea , 9.2 nausea and vomiting, $8.3 \%$ indicated other symptoms, 8.1 had chest pain, 5.2 had joint pain, $4 \%$ had abdominal pain and $2 \%$ had irritability and confusion.

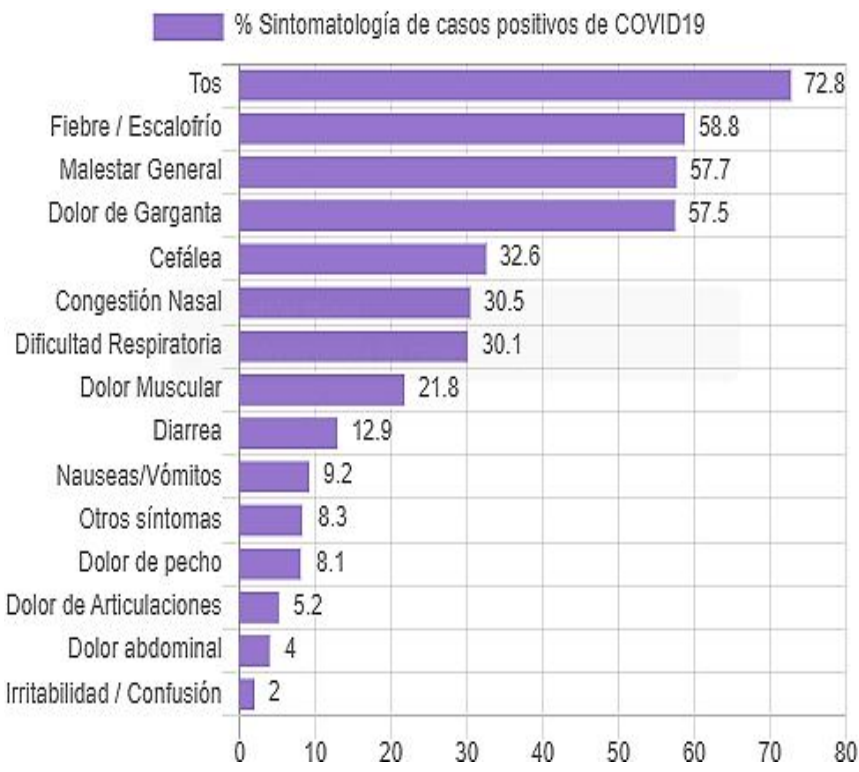

Figure 4:Symptoms of positive covid-19 cases in Peru

\section{F. Data analysis (Use of the decition tree)}

In this part of the figure 5, will analyze existing data of a possible coronavirus symptom through the decision tree that will help with the prediction.

In the descriptive analysis: we can say that through the data and the decision tree shown in Figure 5, and questions showing the symptoms presented by patients infected with the virus earlier that if they now have a high rating according to the decision tree it means that it is very likely that it is infected with the virus

In the diagnostic analysis: according to the analysis shown lines above, it tells us that for a person to become infected with the virus must have direct touch with the individual infected through the airways.

In predictive analysis: it is given thanks to the past data of symptoms of patients confirmed with the virus as shown in Fig. 6 where it allows us to analyze the decision tree in which we can say that if a patient has symptoms of cough, fever, chilled, general discomfort, sore throat, headache, nasal congestion, shortness of breath and muscle aches has an $80 \%$ chance of having or having the COVID_19 virus again. In addition, if a patient counts are the symptoms Medium that are: diarrhea, vomiting, chest pain or other symptoms has a $50 \%$ chance of having the virus. Finally if a patient has symptoms at low level that are: joint pain, abdominal pain and irritability has a $10 \%$ chance of having the virus in their body.

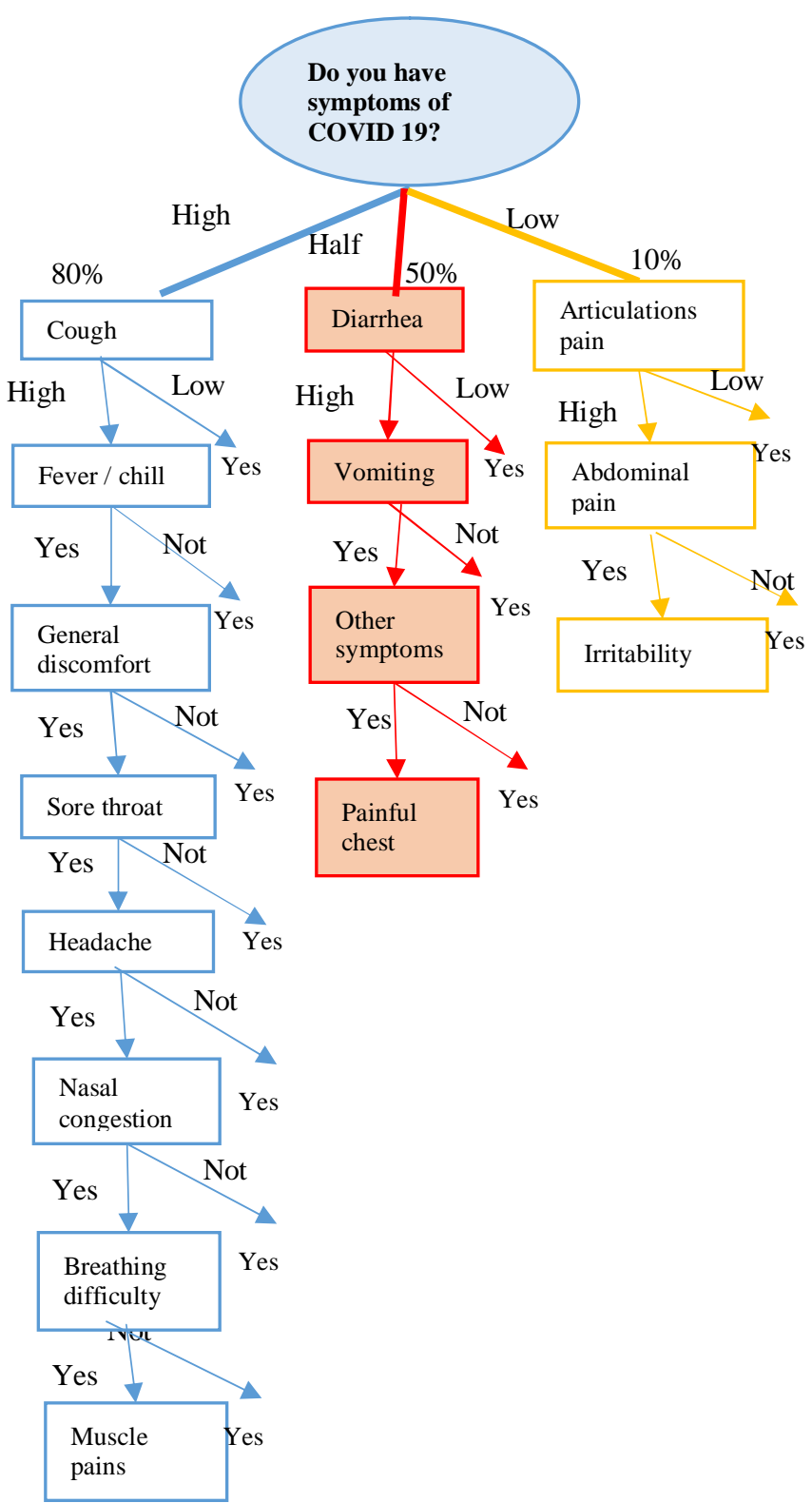

Figure 5:Tree of prediction decideds if a patient has COVID-19

\subsection{Mobile application prototype}

As a result of the case study, the design of a mobile application that has been carried out with a previous analysis for the proper functioning of the application for the operation of the application raised in this work is presented, in this way a series of established stages and functionalities will be achieved, which allow the user to make use of it. MockFLOW, an interactive and easy software for drawings and App interfaces, was used for them.

As a result in this case study, the design of a mobile application that has been carried out with a previous analysis for the proper functioning of the application for the operation of the application raised in this work is presented, in this way a series of established stages and functionalities will be achieved, which allow the user to make use of it. 
MockFLOW, an interactive and easy software for drawings and App interfaces, was used for them.

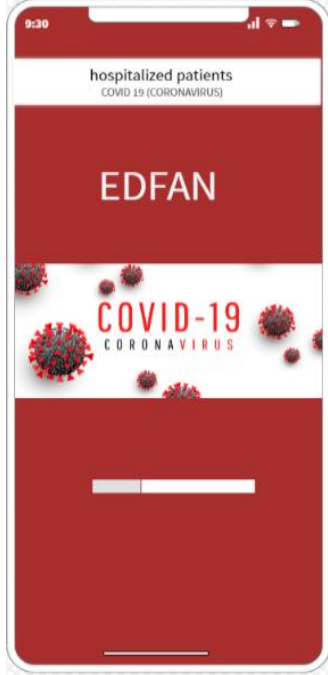

Figure 7: Splash

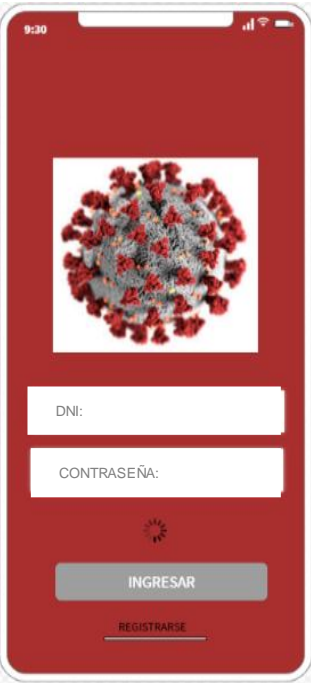

Figure 8: Login
Figure 7 shows the Splash where it will deploy while loading the application, thereby to the user that the app is loading. Figure 8 shows the Login where it is proposed to validate the data of the previously registered user, for this you will be asked for the identity document (ID) and password for greater security of the personal data of the person in the mobile application, in case the user is not registered will be asked to register in order to access the application..

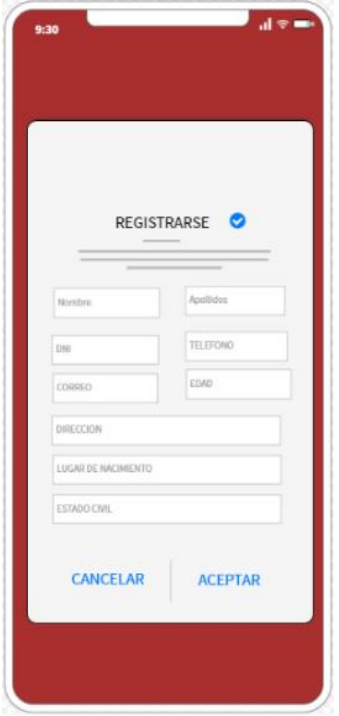

Figure 9: Register

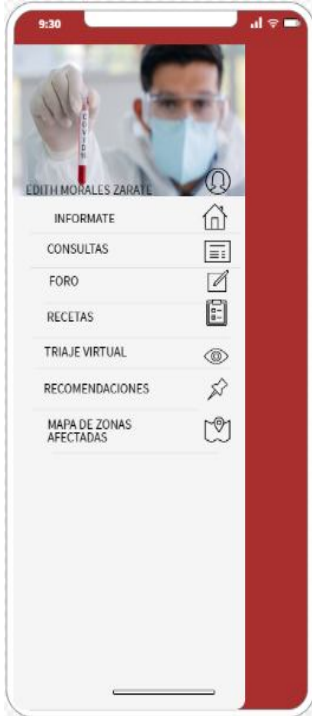

Figure 10: Menu
Figure 9, shows the Registry where the user will fill in all the fields necessary to be able to start the application, this record consists of the personal data such as Name, surname, ID, password, Phone, Address, Date Birth and Civil Status and the registration will start.

Figure 10, shows the application menu, where you have multiple options so that you can customize or configure the platform, as well as query different data. In this option we can display the name of the registered user with the following options: a) Informant: will show general data of up-to-date information regarding COVID_19, the victim, ICU patients, discharged and deceased patients. Inquiries: a medium online chat whereby the patient could make more personal consultations with the doctor online, such as scheduled appointments, personal prescriptions or other required queries as shown in Figure 13.

b) Forums: In this space of the App, it is proposed that users ask questions and answers that are discussed among them to share experiences, suggestions or others by people who live or lived closely the contagion of the virus as a topic of prevention.

c) Prescriptions: In this option, it is suggested that discharged, asymptomatic or potentially contagious patients be informed of the prescriptions for generic medicines, used to combat possible symptoms of coronavirus, as established by the Ministry of Health (MINSA) in an updated form.

d) Virtual triage(discarded tes): In this option is what gives value to the present research work, since by means of which it was proposed to apply machine learning, in which a patient discharged or others, present symptoms of COVID_19, will be presented with a series of questions shown in Figure 11, which have already been analyzed internally where they will determine the possibility of the virus contracting the virus , with a percentage shown in the decition tree proposed in the methodology of the precent.

e) Recommendations: This option proposes that physicians may make recommendations not to spread or be spread by the virus, according to standards established by the World Health Organization (WHO).

f) Map of affected areas: A means by which areas of the country will be shown and identified with the most likely cases of coronavirus infections shown, so that people can take better precautions when moving.

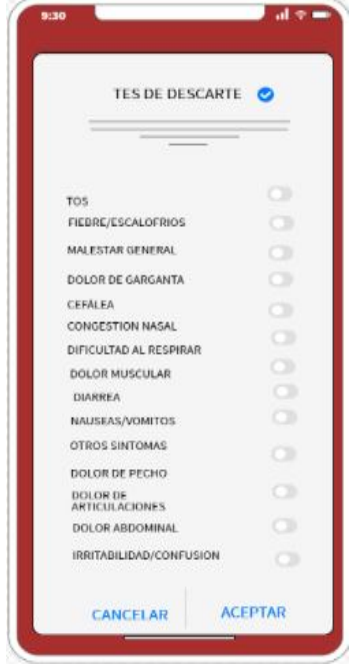

Figure 11:Discard teas

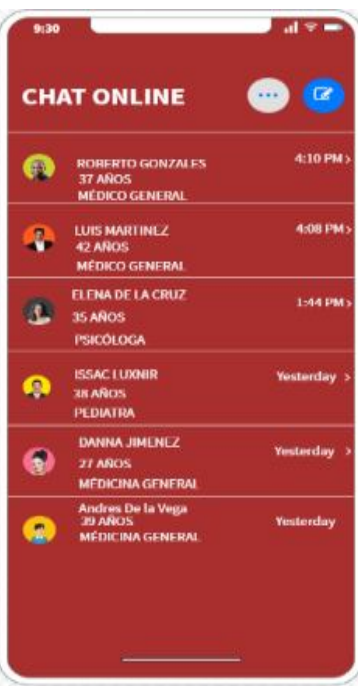

Figure 13: Chat 
Alexi Delgado et al., International Journal of Emerging Trends in Engineering Research, 8(9), September 2020, 6196 - 6202

\section{RESULTS AND DISCUSSION}

The results of the method and case study in this article present the prototype posed and analyzed of what the functionality of the application would look like and the tools that would be used to carry it out compared to other research already implemented; in the part of the methodology will also show as results the comparison of this research with other already validated articles that used Machine Learning for its execution.

\subsection{About the case study}

As for the software proposed in the present prototype, Android is found with Kotlin as a tool that will serve us to convert the Java code into Kotlin so that the source code is reduced and provides other competitive advantages for the development of the App, in other research carried out on the maintainability and modularity of Android mobile applications with anti-pattern and model-view-presenter design pattern, you can see that they applied design patterns that helped them with the reduction of source code and interactivity at the time of programming the application[20]. In addition, another article mentions that object-oriented architecture, for the development of an application, does not guarantee dependency on programming modules, so it is proposed to use a programming language such as Java or $\mathrm{C}++$ in the Model View and Controller (MVC) environment [21].For the interactivity and handling of the application for all types of users, it was proposed to use the Framework such as HTML, JavaScript, JQuery, to show a friendly environment, manageable for all types of user subscribed in the application it is necessary to make interactive applications and easy to understand when training in the behavior of the same[22].

\subsection{About the Methodology}

As the results of the methodology presented in this paper, it can be mentioned that it was very applicable to the topic of predictive analysis of the historical data of the COVID_19 shown lines above, where we can mention that thanks to the correct use of the phases of the ML methodology, the decision tree and the correct programming of the application, the latter will be able to predict whether the patient has or will have coronavirus again according to their symptoms presented, in addition to showing many other functionalities for patient tracking. Then the USE OF ML will allow us that the application can learn the behaviors performed and predict from it various results.

In other research conducted with the use of Machine Learning, it was applied in the topic of patient behavior tracking through the use of video, machine learning and $5 \mathrm{G}$ technology, for monitoring different types of pains through the behavior of the eyes, mouth and agitation status of a patient, in order to evaluate the patient's condition[23]; like the end of the use of machine learning in the present work which is the prediction from past events, in the aforementioned research tells us that it was able to analyze and predict from an easy scan in which condition the patient finds himself and give instant results thanks to events already learned by the system previously. In addition, another research conducted a daily cardiac health monitoring study with the use of EAR-ECG and Machine Learning, using ear ECG datasets acquired by a portable prototype for learning their research. A study was also conducted using ML, for the simulation of the system of a cloud server where they demonstrated the energy saving by downloading computing [24]. Other research also conducted a mobile application for skin cancer screening, where they used cancer dataset and images to the skin to demonstrate and test concepts through machine learning [12].

\section{CONCLUSIONS}

The design of this mobile application for the follow-up of patients discharged from Peru was made to prevent patients with symptoms of COVID_19 from being transferred to a health centre more frequently so that they can infect or be infected. The application was designed for a continuous follow-up at the time a patient is conducive to it as it would have an automatic learning. Also, the efficiency of the application design was demonstrated with the use of MOCKFLOW.

Regarding the methodology, Machine Learning was applied as a method that allowed to improve the understanding and learning of the subject of study, thanks to the great amount of data that could be used. This allowed the identification of behavioural patterns in the updated data.

Finally, with the development of the present in the future, we intend to develop a mobile application with the given indications. In addition, the Machine Learning method used in this research could be useful to carry out different types of studies in which machine learning is required, in order to obtain an informative classification and study the behaviour of the mass data in the future.

\section{REFERENCES}

1. J. M. Molina et al., "No evidence of rapid antiviral clearance or clinical benefit with the combination of hydroxychloroquine and azithromycin in patients with severe COVID-19 infection," Med. Mal. Infect., vol. 50, no. 4, p. 384, 2020, doi: 10.1016/j.medmal.2020.03.006.

2. A. Delgado, A. Aguirre, E. Palomino, G. Salazar, "Applying triangular whitenization weight functions to assess water quality of main affluents of Rimac river,"Proceedings of the 2017 Electronic Congress, E-CON UNI 2017, 2018-January, pp. 1-4.

3. Cámara de Comercio de Lima, "Propuestas Contra el COVID-19," p. 17, 2020, [Online]. Available: https://www.camaralima.org.pe/repositorioaps/0/0/par/e dicion922/la_camara 922_final.pdf.

4. L. Oms and O. Medio, "Atención en el domicilio a pacientes presuntamente infectados por el nuevo 
coronavirus ( COVID-19 ) que presentan síntomas leves , y gestión de sus contactos" pp. 1-5, 2020.

5. A. Picu and T. Spyropoulos, "DTN-Meteo: Forecasting the performance of DTN protocols under heterogeneous mobility," IEEE/ACM Trans. Netw., vol. 23, no. 2, pp. 587-602, 2015, doi: 10.1109/TNET.2014.2301376.

6. M. M. Navarro and B. B. Navarro, "Optimal Short-Term Forecasting Using GA-Based Holt-Winters Method," pp. 681-685, 2019.

7. S. Ray, "A Quick Review of Machine Learning Algorithms," Proc. Int. Conf. Mach. Learn. Big Data, Cloud Parallel Comput. Trends, Prespectives Prospect. Com. 2019, pp. 35-39, 2019, doi: 10.1109/COMITCon.2019.8862451.

8. C. R. Arias, R. M. Duron, and A. V. Delgado-Escueta, "Identification of New Epilepsy Syndromes using Machine Learning,pp. 1-4, 2020, doi: 10.1109/concapanxxxix47272.2019.8977043.

9. A. Delgado, P. Montellanos, J. Llave, "Air quality level assessment in Lima city using the grey clustering method," IEEE ICA-ACCA 2018 - IEEE International Conference on Automation/23rd Congress of the Chilean Association of Automatic Control: Towards an Industry 4.0 - Proceedings, 8609699.

10. [F. Monteiro-Guerra, O. Rivera-Romero, V. Mylonopoulou, G. R. Signorelli, F. Zambrana, and L. Fernandez-Luque, "The Design of a Mobile App for Promotion of Physical Activity and Self-Management in Prostate Cancer Survivors: Personas, Feature Ideation and Low-Fidelity Prototyping," Proc. - IEEE Symp. Comput. Med. Syst., vol. 2017-June, pp. 761-766, 2017, doi: 10.1109/CBMS.2017.75.

11. K. Pahwa and N. Agarwal, "Stock Market Analysis using Supervised Machine Learning,' Proc. Int. Conf. Mach. Learn. Big Data, Cloud Parallel Comput. Trends, Prespectives Prospect. Com. 2019, pp. 197-200, 2019, doi: 10.1109/COMITCon.2019.8862225.

12. X. Dai, I. Spasic, B. Meyer, S. Chapman, and F. Andres, "Machine learning on mobile: An on-device inference app for skin cancer detection," 2019 4th International Conference on Fog and Mobile Edge Computing, FMEC 2019. pp. 301-305, 2019, doi: 10.1109/FMEC.2019.8795362.

13. R. A. Kulkarni, "Scrutinizing action performed by user on mobile app through network using machine learning techniques: A survey," Proc. 2nd Int. Conf. Inven. Syst. Control. ICISC 2018, no. Icisc, pp. 860-863, 2018, doi: 10.1109/ICISC.2018.8398921.

14. N. Gnoza and M. Barberena, 'Estudio de factibilidad del uso de Machine Learning con múltiples fuentes de datos en el pronóstico del tiempo.," 2018.

15. M. Leeds and T. Atkison, "Preliminary Results of Applying Machine Learning Algorithms to Android Malware Detection," Proc. - 2016 Int. Conf. Comput. Sci. Comput. Intell. CSCI 2016, pp. 1070-1073, 2017, doi: 10.1109/CSCI.2016.0204.

16. D. A. Grilo, "Diseño e Implementación de un Sistema basado en Android y Bases de Datos NoSQL para el
Seguimiento de Pacientes," Univ. politécnica Val., pp. $1-52,2015$.

17. Meeradevi and H. Salpekar, "Design and Implementation of Mobile Application for Crop Yield Prediction using Machine Learning," pp. 1-6, 2020, doi: 10.1109/gcat47503.2019.8978315.

18. M. I. Nielsen P. and V. Villarreal, "Diseño y desarrollo de una aplicación móvil que facilite el seguimiento y control de pacientes hipertensos en Panamá,' Memorias Congr. UTP, vol. 0, no. 0, pp. 54-62, 2017, [Online]. Available: http://revistas.utp.ac.pa/index.php/memoutp/article/view /1471.

19. D. K. Bonilla-Aldana, W. E. Villamil-Gómez, A. A. Rabaan, and A. J. Rodríguez-Morales, "A new viral zoonosis of global concern: Coronavirus COVID-19 disease in 2019," Iatreia, vol. 33, no. 2, pp. 107-110, 2020.

20. G. Prabowo, H. Suryotrisongko, and A. Tjahyanto, "A Tale of Two Development Approach: Empirical Study on the Maintainability and Modularity of Android Mobile Application with Anti-Pattern and Model-View-Presenter Design Pattern," Proc. - 2nd 2018 Int. Conf. Electr. Eng. Informatics, ICELTICs 2018, pp. 149-154, 2018, doi: 10.1109/ICELTICS.2018.8548784.

21. V. N. Inukollu, T. Kang, and N. Sakhnini, "Design constraints and challenges behind fault tolerance systems in a mobile application framework," Proceeding 2015 10th Int. Des. Test Symp. IDT 2015, pp. 159-160, 2016, doi: 10.1109/IDT.2015.7396760.

22. M. Esteves and A. Pereira, "Y.S.Y.D. - You stay you demand: User-centered design approach for mobile hospitality application,” Proc. 2015 Int. Conf. Interact. Mob. Commun. Technol. Learn. IMCL 2015, no. November, pp. 318-322, 2015, doi: 10.1109/IMCTL.2015.7359611.

23. A. Delgado, I. Romero, "Applying the Grey Systems Theory to Assess Social Impact from an Energy Project,'Proceedings of the 2018 IEEE 25th International Conference on Electronics, Electrical Engineering and Computing, INTERCON 2018, 8526372.

24. S. M. Azharul Karim and J. J. Prevost, "A machine learning based approach to mobile cloud offloading," Proc. Comput. Conf. 2017, vol. 2018-Janua, no. July 2017, pp. 675-680, 2018, doi: 10.1109/SAI.2017.8252168. 